WOJCIECH M. ZAJĄCZKOWSKI (Warszawa)

\title{
ON IMBEDDING THEOREMS FOR WEIGHTED ANISOTROPIC SOBOLEV SPACES
}

Abstract. Using the Il'in integral representation of functions, imbedding theorems for weighted anisotropic Sobolev spaces in $\mathbb{E}^{n}$ are proved. By the weight we assume a power function of the distance from an $(n-2)$-dimensional subspace passing through the domain considered.

1. Introduction. The aim of this paper is to show some imbedding theorems for weighted Sobolev spaces. We introduce the weighted Sobolev spaces $W_{p, \mu}^{l, l / 2}\left(\Omega^{T}\right), l=2 k, k \in \mathbb{N} \cup\{0\}, \Omega \subset \mathbb{E}^{n}, \Omega^{T}=\Omega \times(0, T), \mu \in \mathbb{R}$, $p \geq 1$, with the norm

$$
\|u\|_{W_{p, \mu}^{l, l / 2}\left(\Omega^{T}\right)}=\left(\sum_{|\alpha|+2 \alpha_{0} \leq l} \int_{\Omega^{T}}\left|D_{x}^{\alpha} \partial_{x_{0}}^{\alpha_{0}} u\right|^{p} \varrho^{p \mu}(x) d x\right)^{1 / p},
$$

where $\alpha=\left(\alpha_{1}, \ldots, \alpha_{n}\right)$ is a multiindex, $|\alpha|=\alpha_{1}+\ldots+\alpha_{n}, D_{x}^{\alpha}=\partial_{x_{1}}^{\alpha_{1}} \ldots \partial_{x_{n}}^{\alpha_{n}}$ and $\varrho=\varrho(x)$ is the distance from $x$ to either a subspace of $\mathbb{R}^{n}$ or a point. In this paper we assume that $\varrho(x)=\operatorname{dist}(x, M)$ where $M$ is an $(n-2)$ dimensional subspace of $\mathbb{E}^{n}$. To simplify considerations we assume that $M$ is defined by $x_{1}=x_{2}=0$. Finally $\mathbb{E}^{n}$ is the $n$-dimensional Euclidean space. Therefore, we can assume that $\varrho(x)=\sqrt{x_{1}^{2}+x_{2}^{2}}$.

More precisely we define $W_{p, \mu}^{l, l / 2}\left(\Omega^{T}\right)$ as the closure of the set $C_{0}^{\infty}\left(\Omega^{T} \backslash M\right)$ in the norm (1.1).

We use the standard anisotropic notation (see [1]). We consider more general anisotropic Sobolev spaces $W_{p, \mu}^{\bar{l}}\left(\mathbb{E}^{n+1}\right)$, where $\bar{l}=\left(l_{0}, l_{1}, \ldots, l_{n}\right)$,

2000 Mathematics Subject Classification: Primary 46E35.

Key words and phrases: weighted Sobolev spaces, imbedding theorems, anisotropic Sobolev spaces.

Research supported by the Polish KBN Grant No. 2 P03A-038-16. 
with the norm defined as follows:

$$
\begin{aligned}
\|u\|_{W_{p, \mu}^{\bar{l}}\left(\mathbb{E}^{n+1}\right)} & =\|u\|_{L_{p, \mu}\left(\mathbb{E}^{n+1}\right)}+\sum_{i=0}^{n}\left\|\partial_{x_{i}}^{l_{i}} u\right\|_{L_{p, \mu}\left(\mathbb{E}^{n+1}\right)} \\
& \equiv\|u\|_{L_{p, \mu}\left(\mathbb{E}^{n+1}\right)}+\|u\|_{L_{p, \mu}^{\bar{l}}\left(\mathbb{E}^{n+1}\right)},
\end{aligned}
$$

where $\|u\|_{L_{p, \mu}\left(\mathbb{E}^{n+1}\right)}=\left(\int_{\mathbb{E}^{n+1}}|u|^{p} \varrho^{p \mu} d x\right)^{1 / p}$. Comparing (1.1) and (1.2) we see that for a norm equivalent to (1.1) we have

$$
l_{0}=l / 2, \quad l_{i}=l, \quad i=1, \ldots, n .
$$

We also introduce $\bar{\sigma}=\left(\sigma_{0}, \sigma_{1}, \ldots, \sigma_{n}\right)$ and $\sigma_{i}=1 / l_{i}, i=0,1, \ldots, n$. In the case of the norm (1.1) we have

$$
\sigma_{0}=2 / l, \quad \sigma_{i}=1 / l, \quad i=1, \ldots, n .
$$

We use the following integral representation of a function $f$ with integrable $\bar{l}$-derivative (see $[1,3]$ ):

$$
f(\bar{x})=f_{r^{\bar{\sigma}}}(\bar{x})+\int_{0}^{r} \sum_{i=0}^{n} \int_{\mathbb{E}^{n+1}} h^{-|\bar{\sigma}|} \Phi_{i}\left(\bar{x} / h^{\bar{\sigma}}\right) D_{i}^{l_{i}} f(\bar{x}+\bar{y}) d \bar{y} d h,
$$

where $\bar{x}=\left(x_{0}, x_{1}, \ldots, x_{n}\right), \bar{y}=\left(y_{0}, y_{1}, \ldots, y_{n}\right), x_{0}=t, y_{0}=\tau,|\bar{\sigma}|=\sigma_{0}+$ $\sum_{i=1}^{n} \sigma_{i}, h^{\bar{\sigma}}=\left(h^{\sigma_{0}}, h^{\sigma_{1}}, \ldots, h^{\sigma_{n}}\right), \bar{y} / h^{\bar{\sigma}}=\left(y_{0} / h^{\sigma_{0}}, y_{1} / h^{\sigma_{1}}, \ldots, y_{n} / h^{\sigma_{n}}\right), D_{i}$ denotes the derivative with respect to the $i$ th argument, and

$$
f_{r^{\bar{\sigma}}}(\bar{x})=r^{-|\bar{\sigma}|} \int_{\mathbb{E}^{n+1}} \Phi_{*}\left(\bar{y} / r^{\bar{\sigma}}\right) f(\bar{x}+\bar{y}) d \bar{y} .
$$

We assume that $\Phi_{*}, \Phi_{i} \in C_{0}^{\infty}\left(\mathbb{E}^{n+1}\right), i=0,1, \ldots, n$, have compact supports in the first coordinate angle and for any $\alpha$,

$$
\int D^{\alpha} \Phi_{i}(\bar{x}) d \bar{x}=0, \quad i=0,1, \ldots, n, \quad \int D^{\alpha} \Phi_{*}(\bar{x}) d \bar{x}=0 .
$$

Moreover, we assume that the supports of $\Phi_{*}, \Phi_{i}, i=0,1, \ldots, n$, belong to the "horn" (see $[1,3])$

$$
\begin{aligned}
R(\bar{l}, r, \varepsilon)=\left\{\bar{y}: y_{i}>0, a_{i}>0,0\right. & <a_{i} h<y_{i}^{l_{i}}<\left(a_{i}+\varepsilon\right) h, \\
i & =0,1, \ldots, n, 0<h<r<\infty\},
\end{aligned}
$$

where $\varepsilon>0$. If $l_{i}=l, i=0,1, \ldots, n$, the horn $R(\bar{l}, r, \varepsilon)$ becomes the cone $V(r, \varepsilon)=\left\{y: y_{i}>0, a_{i}>0,0<a_{i} h<y_{i}<\left(a_{i}+\varepsilon\right) h, i=0,1, \ldots, n, 0<\right.$ $h<r<\infty\}$. For simplicity we shall omit the $\varepsilon$ in $R(\bar{l}, r, \varepsilon)$ and $V(r, \varepsilon)$.

We define

$$
f_{\varepsilon}(\bar{x})=f_{r^{\bar{\sigma}}}(\bar{x})+\int_{\varepsilon}^{r} d h \sum_{i=0}^{n} \int_{\mathbb{E}^{n+1}} h^{-|\bar{\sigma}|} \Phi_{i}\left(\bar{y} / h^{\bar{\sigma}}\right) D_{i}^{l_{i}} f(\bar{x}+\bar{y}) d \bar{y} .
$$


From [3] we have the estimate

$$
\int_{\varepsilon}^{r} h^{\tau-1} \Phi_{i}^{(\bar{\nu}(k))}(y / h) d h \leq c \chi(y, R(\bar{l}, r))\left(\sum_{i=0}^{n}\left|y_{i}\right|^{l_{i}}\right)^{\tau}
$$

where $\chi(y, R(\bar{l}, r))$ is the characteristic function of the horn $R(\bar{l}, r)$ and $\Phi_{i}^{(\bar{\nu}(k))}$ is defined below (2.3).

For the reader's convenience we recall some results. From [3] we recall the following extension of the Calderón-Zygmund theorem.

Lemma 1.1. Let the support of $\Phi \in C_{0}^{\infty}\left(\mathbb{E}^{n}\right)$ be in the first coordinate cube and $\int_{\mathbb{E}^{n}} \Phi(x) d x=0$. Let $1<p<\infty$ and let

$$
v_{\varepsilon r}(x)=\int_{\varepsilon}^{r} d h \int_{\mathbb{E}^{n}} h^{-1-|\sigma|} \Phi\left(y / h^{\sigma}\right) u(x+y) d y
$$

for $u \in L_{p}\left(\mathbb{E}^{n}\right), x, y \in \mathbb{E}^{n}, \sigma \in \mathbb{R}_{+}$. Then

$$
\left\|v_{\varepsilon r}\right\|_{L_{p}\left(\mathbb{E}^{n}\right)} \leq c_{p}\|u\|_{L_{p}\left(\mathbb{E}^{n}\right)}
$$

and

$$
v_{\varepsilon r} \rightarrow v_{0 r} \quad \text { in } L_{p}\left(\mathbb{E}^{n}\right) \text { as } \varepsilon \rightarrow 0 .
$$

We also need the Hardy inequality

$$
\left\||x|^{-\gamma} F(x)\right\|_{L_{p}\left(\mathbb{E}_{+}\right)} \leq c\left\||x|^{-\gamma+1} f(x)\right\|_{L_{p}\left(\mathbb{E}_{+}\right)}, \quad 1 \leq p \leq \infty,
$$

where $\gamma \neq 1 / p, F(x)=\int_{x}^{\infty} f(\xi) d \xi$ for $\gamma<1 / p, F(x)=\int_{0}^{x} f(\xi) d \xi$ for $\gamma>1 / p$ and $\mathbb{E}_{+}=\{x \in \mathbb{E}: x>0\}$.

In the case of isotropic weighted Sobolev spaces similar results are proved in $[4]$.

From $[1$, Ch. 2, Sect. 8] we recall

Definition 1.2. We say that a domain $Q$ satisfies that the $R(\bar{l}, r)$-horn condition if there exist $K$ open subdomains $Q_{k}$ and horns $R_{k}(\bar{l}, r)$ such that

$$
Q=\bigcup_{k=1}^{K} Q_{k}=\bigcup_{k=1}^{K}\left(Q_{k}+R_{k}(\bar{l}, r)\right) .
$$

In [1] this property is called the weak $R(\bar{l}, r)$-horn condition.

2. Imbedding theorems for $p \neq q$. First we prove

Theorem 2.1. Assume that $f \in W_{p, \alpha}^{\bar{l}}(Q), Q \subset \mathbb{E}^{n+1}, \bar{l}=\left(l_{0}, l_{1}, \ldots, l_{n}\right)$, $1<p<q<\infty, \alpha, \beta \in \mathbb{R}_{+}, 0<l_{i} \in \mathbb{Z}, 0 \leq \nu_{i} \in \mathbb{Z}, i=0,1, \ldots, n$, $l_{1}=l_{2}=l_{*}$,

$$
\varkappa=1-\left(\frac{1}{p}-\frac{1}{q}\right) \sum_{i=0}^{n} \frac{1}{l_{i}}-\sum_{i=0}^{n} \frac{\nu_{i}}{l_{i}}-\frac{1}{l_{*}}(\alpha-\beta) \geq 0,
$$


where $Q$ satisfies the $R(\bar{l}, r)$-horn condition. Assume

$$
\alpha>\beta \text {. }
$$

Then $D^{\bar{\nu}} f \in L_{q, \beta}(Q)$ and

$$
\left\|D^{\bar{\nu}} f\right\|_{L_{q, \beta}(Q)} \leq c_{1} \delta^{\varkappa}\|f\|_{L_{p, \alpha}^{\bar{l}}(Q)}+c_{2} \delta^{\varkappa-1}\|f\|_{L_{p, \alpha}(Q)},
$$

where the constants $c_{1}, c_{2}$ do not depend on $f, \delta \in\left(0, h_{0}\right), h_{0}=h_{0}(Q)$.

Proof. Let $x=\left(x_{1}, \ldots, x_{n}\right), x^{\prime}=\left(x_{1}, x_{2}\right), x^{\prime \prime}=\left(x_{3}, \ldots, x_{n}\right)$. Then we introduce the cylindrical coordinates $\left(\varrho_{x}, \varphi_{x}, x^{\prime \prime}\right)$ connected with $x$, where $\varrho_{x}=\left|x^{\prime}\right|, x_{1}=\varrho_{x} \cos \varphi_{x}, x_{2}=\varrho_{x} \sin \varphi_{x}$.

Let $k \in \mathbb{N}_{0}$. Then integrating by parts and using the compactness of the supports of $\Phi_{*}, \Phi_{i}, i=0,1, \ldots, n$, we obtain from (1.7) the expression

$$
\begin{aligned}
& D^{\bar{\nu}} f_{\varepsilon}(\bar{x}) \\
& =c \int_{\mathbb{E}^{n+1}} \Phi_{*}^{(\bar{\nu}(k))}\left(\bar{y} / r^{\bar{\sigma}}\right) \int_{\varrho_{y}}^{\infty} \int_{t_{k-1}}^{\infty} \ldots \int_{t_{1}}^{\infty} f\left(\bar{x}+\bar{y}\left(t_{0}\right)\right) d t_{0} d t_{1} \ldots d t_{k-1} d \bar{y} \\
& \quad+\int_{\varepsilon}^{r} d h \sum_{i=0}^{n} \int_{\mathbb{E}^{n+1}} h^{-|\bar{\sigma}|-k \sigma_{*}-(\bar{\sigma}, \bar{\nu})} \Phi_{i}^{(\bar{\nu}(k))}\left(\bar{y} / h^{\bar{\sigma}}\right) \\
& \quad \times \int_{\varrho_{y}}^{\infty} \int_{t_{k-1}}^{\infty} \ldots \int_{t_{1}}^{\infty} D_{i}^{l_{i}} f\left(\bar{x}+\bar{y}\left(t_{0}\right)\right) d t_{0} \ldots d t_{k-1} d \bar{y},
\end{aligned}
$$

where $\sigma_{*}=\sigma_{1}=\sigma_{2}$ so that $l_{1}=l_{2}=l_{*}, \bar{\nu}=\left(\nu_{0}, \nu_{1}, \ldots, \nu_{n}\right), \bar{\nu}(k)=$ $\left(\nu_{0}, \nu_{1}+k_{1}, \nu_{2}+k_{2}, \nu_{3}, \ldots, \nu_{n}\right), k_{1}+k_{2}=k$,

$\Phi^{(\bar{\nu}(k))}(x)=\sum_{k_{1}+k_{2}=k} c_{k_{1} k_{2}}\left(\cos \varphi_{x}\right)^{k_{1}}\left(\sin \varphi_{x}\right)^{k_{2}} \partial_{x_{0}}^{\nu_{0}} \partial_{x_{1}}^{\nu_{1}+k_{1}} \partial_{x_{2}}^{\nu_{2}+k_{2}} \partial_{x_{3}}^{\nu_{3}} \ldots \partial_{x_{n}}^{\nu_{n}} \Phi(x)$, $\bar{y}=\left(y_{0}, y_{1}, \ldots, y_{n}\right), \bar{y}\left(t_{0}\right)=\left(y_{0}, t_{0} \cos \varphi_{y}, t_{0} \sin \varphi_{y}, y_{3}, \ldots, y_{n}\right)$, and $(\bar{\sigma}, \bar{\nu})=$ $\sigma_{0} \nu_{0}+\sigma_{1} \nu_{1}+\ldots+\sigma_{n} \nu_{n}$. Finally $c_{k_{1} k_{2}}$ are determined by the relation

$$
\partial_{\varrho_{x}}^{k} \Phi=\sum_{k_{1}+k_{2}=k} c_{k_{1} k_{2}}\left(\frac{\partial x_{1}}{\partial \varrho_{x}}\right)^{k_{1}}\left(\frac{\partial x_{2}}{\partial \varrho_{x}}\right)^{k_{2}} \partial_{x_{1}}^{k_{1}} \partial_{x_{2}}^{k_{2}} \Phi .
$$

Let us introduce the notation

$$
\begin{aligned}
F(\bar{y}) & =\int_{\varrho_{y}}^{\infty} \int_{t_{k-1}}^{\infty} \ldots \int_{t_{1}}^{\infty} f\left(\bar{y}\left(t_{0}\right)\right) d t_{0} d t_{1} \ldots d t_{k-1} \\
F_{i}(\bar{y}) & =\int_{\varrho_{y}}^{\infty} \int_{t_{k-1}}^{\infty} \ldots \int_{t_{1}}^{\infty} D_{i}^{l_{i}} f\left(\bar{y}\left(t_{0}\right)\right) d t_{0} d t_{1} \ldots d t_{k-1} .
\end{aligned}
$$


Then we can write (2.3) in the following shorter form:

$$
\begin{aligned}
D^{\bar{\nu}} f_{\varepsilon}(\bar{x}) & =c \int_{\mathbb{E}^{n+1}} \Phi^{(\bar{\nu}(k))}\left(\bar{y} / r^{\bar{\sigma}}\right) F(\bar{x}+\bar{y}) d \bar{y} \\
& +c \int_{\varepsilon}^{r} d h \sum_{i=0}^{n} \int_{\mathbb{E}^{n+1}} h^{-|\bar{\sigma}|-k \sigma_{*}-(\bar{\sigma}, \bar{\nu})} \Phi_{i}^{(\bar{\nu}(k))}\left(\bar{y} / h^{\bar{\sigma}}\right) F_{i}(\bar{x}+\bar{y}) d \bar{y} .
\end{aligned}
$$

Now we examine the case $\varkappa=0$ (see (2.1)). Consider

$$
\begin{aligned}
\left\|D^{\bar{\nu}} f_{\varepsilon}(\bar{x})\right\|_{L_{q, \beta}(Q) \leq} & c\left\|\int_{\mathbb{E}^{n+1}} \Phi_{*}^{(\bar{\nu}(k))}\left(\bar{y} / r^{\bar{\sigma}}\right) F(\bar{x}+\bar{y}) d \bar{y}\right\|_{L_{q, \beta}(Q)} \\
& +c \sum_{i=0}^{n} \| \int_{\varepsilon}^{r} d h \int_{\mathbb{E}^{n+1}} h^{-|\bar{\sigma}|-k \sigma_{*}-(\bar{\sigma}, \bar{\nu})} \Phi_{i}^{(\bar{\nu}(k))}\left(\bar{y} / h^{\bar{\sigma}}\right) \\
& \times \frac{\left|x^{\prime}\right|^{\beta}}{\left|x^{\prime}+y^{\prime}\right|^{\alpha-k}} F_{i}^{\prime}(\bar{x}+\bar{y}) d \bar{y} \|_{L_{q}(Q)} \equiv M+N,
\end{aligned}
$$

where $F_{i}^{\prime}(x)=\left|x^{\prime}\right|^{\alpha-k} F_{i}(x)$ and $\left|x^{\prime}\right|=\sqrt{x_{1}^{2}+x_{2}^{2}}$.

First we estimate $N$. Using (1.8) we obtain

$$
\begin{aligned}
N \leq c \sum_{i=0}^{n} \| \int_{\mathbb{E}^{n+1}} \chi\left(y, R\left(\bar{l}, h_{0}\right)\right) & \left(\sum_{i=0}^{n}\left|y_{i}\right|^{l_{i}}\right)^{\tau} \\
& \times \frac{\left|x^{\prime}\right|^{\beta}}{\left|x^{\prime}+y^{\prime}\right|^{\alpha-k}} F_{i}^{\prime}(\bar{x}+\bar{y}) d \bar{y} \|_{L_{q}\left(\mathbb{E}^{n+1}\right)} \equiv N_{1},
\end{aligned}
$$

where $\tau=-(1-1 / p+1 / q)|\bar{\sigma}|+(\alpha-\beta-k) \sigma_{*}$.

Since $q>p>1$ we can assume that $1 / p-1 / q=1-1 / s$ and we also assume also that $\alpha-\beta-k \leq 1 / s$. To estimate $N_{1}$ we apply the onedimensional Young inequality

$$
\|f * g\|_{L_{q}} \leq\|g\|_{L_{s}}\|f\|_{L_{p}} \quad \text { for } \frac{1}{p}-\frac{1}{q}=1-\frac{1}{s}, 1 \leq p \leq q \leq \infty,
$$

with respect to the variables $x_{0}, x_{3}, \ldots, x_{n}$.

Using (2.8) with respect to $x_{0}$ we find that the kernel in $N_{1}$ is estimated as follows:

$$
\begin{aligned}
& \left(\int_{0}^{\infty}\left(\sum_{i=0}^{n}\left|y_{i}\right|^{l_{i}}\right)^{\tau s} d y_{0}\right)^{1 / s}=\left(\int_{0}^{\infty}\left(\left|y_{0}\right|^{l_{0}}+a_{0}\right)^{\tau s} d y_{0}\right)^{1 / s} \quad\left(a_{0}=\sum_{i=1}^{n}\left|y_{i}\right|^{l_{i}}\right) \\
& \quad \leq\left(\int_{0}^{\infty}\left(y_{0}+a_{0}^{1 / l_{0}}\right)^{\tau s l_{0}} d y_{0}\right)^{1 / s}=\left(\left.\frac{1}{\tau s l_{0}+1}\left(y_{0}+a_{0}^{1 / l_{0}}\right)^{\tau s l_{0}+1}\right|_{y_{0}=0} ^{y_{0}=\infty}\right)^{1 / s} \\
& \quad=\left(-\frac{1}{\tau s l_{0}+1}\right)^{1 / s} a_{0}^{\tau+1 /\left(s l_{0}\right)} \equiv K_{1}
\end{aligned}
$$


where we have used the fact that

$$
\begin{aligned}
\tau s l_{0}+1= & {\left[-\frac{1}{s}\left(\frac{1}{l_{0}}+\frac{1}{l_{1}}+\frac{1}{l_{2}}+\ldots+\frac{1}{l_{n}}\right)+(\alpha-\beta-k) \frac{1}{l_{*}}\right] s l_{0}+1 } \\
= & -l_{0}\left(\frac{1}{l_{1}}+\frac{1}{l_{2}}+\frac{1}{l_{3}}+\ldots+\frac{1}{l_{n}}\right)+\frac{s l_{0}}{l_{*}}(\alpha-\beta-k) \\
& \leq-\frac{2 l_{0}}{l_{*}}+\frac{s l_{0}}{l_{*}}(\alpha-\beta-k)-l_{0}\left(\frac{1}{l_{3}}+\ldots+\frac{1}{l_{n}}\right) \\
& \leq-\frac{l_{0}}{l_{*}}-l_{0}\left(\frac{1}{l_{3}}+\ldots+\frac{1}{l_{n}}\right)<0,
\end{aligned}
$$

since $l_{1}=l_{2}=l_{*}, \alpha-\beta-k \leq 1 / s$.

Using (2.8) with respect to $x_{3}$ we see that the kernel $K_{1}$ is estimated by

$$
\begin{aligned}
\left(\int_{0}^{\infty}\left(\sum_{i=1}^{n}\left|y_{i}\right|^{l_{i}}\right)^{\tau s+1 / l_{0}} d y_{3}\right)^{1 / s} & \leq\left(\int_{0}^{\infty}\left(y_{3}+a_{3}^{1 / l_{3}}\right)^{\left(\tau s+1 / l_{0}\right) l_{3}} d y_{3}\right)^{1 / s} \\
& =\left(-\frac{1}{\tau s l_{3}+l_{3} / l_{0}+1}\right)^{1 / s} a_{3}^{\left[\left(\tau s+1 / l_{0}\right) l_{3}+1\right] /\left(s l_{3}\right)},
\end{aligned}
$$

where $a_{3}=\left|y_{1}\right|^{l_{1}}+\left|y_{2}\right|^{l_{2}}+\sum_{i=4}^{n}\left|y_{i}\right|^{l_{i}}$,

$$
\begin{aligned}
\tau s l_{3}+\frac{l_{3}}{l_{0}} & +1=\left(\tau s+\frac{1}{l_{0}}+\frac{1}{l_{3}}\right) l_{3} \\
= & {\left[-\left(\frac{1}{l_{0}}+\frac{1}{l_{1}}+\frac{1}{l_{2}}+\frac{1}{l_{3}}+\ldots+\frac{1}{l_{n}}\right)+(\alpha-\beta-k) \frac{s}{l_{*}}+\frac{1}{l_{0}}+\frac{1}{l_{3}}\right] l_{3} } \\
\leq & {\left[-\frac{1}{l_{*}}-\left(\frac{1}{l_{4}}+\frac{1}{l_{5}}+\ldots+\frac{1}{l_{n}}\right)\right] l_{3}<0, }
\end{aligned}
$$

where we have used the fact that $l_{1}=l_{2}=l_{*}, \alpha-\beta-k \leq 1 / s$.

Continuing the calculations we obtain

$$
\begin{aligned}
& N_{1} \leq c \sum_{i=0}^{n} \| \int_{\mathbb{E}^{2}} \chi\left(y^{\prime}-x^{\prime}, R\left(l_{*}, h_{0}\right)\right) \mid x^{\prime}-\left.y^{\prime}\right|^{-2 / s+\alpha-\beta-k} \\
& \times \frac{\left|x^{\prime}\right|^{\beta}}{\left|y^{\prime}\right|^{\alpha-k}} G_{i}\left(y^{\prime}\right) d y^{\prime} \|_{L_{q}\left(\mathbb{E}^{2}\right)} \equiv N_{2},
\end{aligned}
$$

where $G_{i}\left(y^{\prime}\right)=\left(\int_{Q}\left|F_{i}^{\prime}(\bar{y})\right|^{p} d y_{0} d y_{3} \ldots d y_{n}\right)^{1 / p}$.

To estimate $N_{2}$ we use Remark 3.1 from [2], which we formulate in the following form. Assume that

$$
p_{1} \geq 1, \quad q_{1} \geq 1, \quad 1 / p_{1}+1 / q_{1} \geq 1, \quad \delta<2 / q_{1}^{\prime}, \quad \lambda<2 / p_{1}^{\prime}+2 / q_{1}^{\prime},
$$


where $r^{\prime}$ is dual to $r$, so $1 / r+1 / r^{\prime}=1$. Then

$$
\begin{aligned}
& \left(\int_{\mathbb{E}^{2}}\left|\frac{1}{|x|^{2 / p_{1}^{\prime}+2 / q_{1}^{\prime}-(\lambda+\delta)}} \int_{|y| \leq|x|} \frac{g(y) d y}{|x-y|^{\lambda}|y|^{\delta}}\right|^{p_{1}^{\prime}} d x\right)^{1 / p_{1}^{\prime}} \leq c\|g\|_{L_{q_{1}}\left(\mathbb{E}^{2}\right)}, \\
& \left(\int_{\mathbb{E}^{2}}\left|\frac{1}{|y|^{\delta}} \int_{|y| \leq|x|} \frac{f(x) d x}{|x-y|^{\lambda}|x|^{2 / p_{1}^{\prime}+2 / q_{1}^{\prime}-(\lambda+\delta)}}\right|^{q_{1}^{\prime}} d y\right)^{1 / q_{1}^{\prime}} \leq c\|f\|_{L_{p_{1}}\left(\mathbb{E}^{2}\right)} .
\end{aligned}
$$

Inserting $p_{1}^{\prime}:=q, q_{1}:=p, \delta:=\delta_{1}$ into (2.10) yields

$$
\left(\int_{\mathbb{E}^{2}}\left|\frac{1}{|x|^{2 / s-\left(\lambda+\delta_{1}\right)}} \int_{|y| \leq|x|} \frac{g(y) d y}{|x-y|^{\lambda}|y|^{\delta_{1}}}\right|^{q} d x\right)^{1 / q} \leq c\|g\|_{L_{p}\left(\mathbb{E}^{2}\right)} .
$$

Inserting $p_{1}:=p, q_{1}^{\prime}:=q, x:=y, y:=x, f:=g, \delta:=\delta_{2}$ in (2.11) implies

$$
\left(\int_{\mathbb{E}^{2}}\left|\frac{1}{|x|^{\delta_{2}}} \int_{|x| \leq|y|} \frac{g(y) d y}{|x-y|^{\lambda}|y|^{2 / s-\left(\lambda+\delta_{2}\right)}}\right|^{q} d x\right)^{1 / q} \leq c\|g\|_{L_{p}\left(\mathbb{E}^{2}\right)} .
$$

The conditions (2.9) imply for (2.12) the restrictions

$$
\delta_{1}<2(1-1 / p), \quad \lambda<2 / s .
$$

For case (2.13) conditions (2.9) yield

$$
\delta_{2}<2 / q, \quad \lambda<2 / s .
$$

Comparing (2.12) with $N_{2}$ we see that

$$
\lambda=\frac{2}{s}-(\alpha-\beta-k), \quad \delta_{1}=\alpha-k, \quad-\beta=\frac{2}{s}-\left(\lambda-\delta_{1}\right),
$$

where the last condition follows from the first two, and comparing (2.13) with $N_{2}$ we obtain

$$
\lambda=\frac{2}{s}-(\alpha-\beta-k), \quad \delta_{2}=-\beta, \quad \alpha-k=\frac{2}{s}-\left(\lambda+\delta_{2}\right),
$$

where the last condition also follows from the first two.

However to estimate $N_{2}$ we need estimate (2.13). Therefore we have to impose the following restrictions:

$$
-\beta<2 / q, \quad 2 / s-(\alpha-\beta-k)<2 / s,
$$

where the last inequality implies

$$
\alpha-k>\beta .
$$

For $k=0$ the condition gives

$$
\alpha>\beta,
$$

so the case $\alpha=\beta$ cannot be considered. Since the first condition of (2.18) is trivial and (2.19) with $k=0$ is less restrictive we see that (2.20) is exactly $\left(2.1^{\prime}\right)$. 
Now using Remark 3.1 from [2] we obtain the estimate

$$
N_{1} \leq c \sum_{i=0}^{n}\left\|F_{i}^{\prime}\right\|_{L_{p}\left(\mathbb{E}^{n+1}\right)} \leq c \sum_{i=0}^{n}\left\|D_{i}^{l_{i}} f\right\|_{L_{p, \alpha}\left(\mathbb{E}^{n+1}\right)},
$$

where in the second inequality we exploited the Hardy inequality.

Similarly using (1.8) we have

$$
M \leq c\left\|F^{\prime}\right\|_{L_{p}\left(\mathbb{E}^{n+1}\right)} \leq c\|f\|_{L_{p, \alpha}\left(\mathbb{E}^{n+1}\right)} .
$$

From (2.21) and (2.22) we obtain (2.2) with $\delta=c, \varkappa=0$ and $Q=\mathbb{E}^{n+1}$ after letting $\varepsilon \rightarrow 0$ (see [3, p. 139]).

To show (2.2) with parameter $\delta$ and $\varkappa>0$ we exploit the considerations from [1, Ch. 3].

To obtain (2.2) for $Q$ bounded we apply the standard considerations with a partition of unity. This concludes the proof.

It seems that condition $\left(2.1^{\prime}\right)$ is artificial. It follows from applying [2] to estimate the integral $N_{2}$. However we do not know how to estimate $N_{2}$ in a different way.

From $(2.1)^{\prime}$ we see that the case

$$
0 \geq \alpha \geq \beta
$$

is not included in Theorem 1. Hence we need

Corollary 2. Assume that $Q$ is bounded and satisfies the $R\left(\bar{l}, h_{0}\right)$-horn condition, $f \in W_{p, \alpha}^{\bar{l}}(Q)$, and

$$
\alpha \leq \beta \text {. }
$$

Take $\alpha^{\prime}>\beta$ such that

$$
\varkappa^{\prime}=1-\left(\frac{1}{p}-\frac{1}{q}\right) \sum_{i=0}^{n} \frac{1}{l_{i}}-\sum_{i=0}^{n} \frac{\nu_{i}}{l_{i}}-\frac{1}{l_{*}}\left(\alpha^{\prime}-\beta\right) \geq 0 .
$$

Then $D^{\bar{\nu}} f \in L_{q, \beta}(Q)$ and

$$
\left\|D^{\bar{\nu}} f\right\|_{L_{q, \beta}(Q)} \leq c \varepsilon^{\varkappa^{\prime}}\|f\|_{L_{p, \alpha}^{\bar{\tau}}(Q)}+c \varepsilon^{\varkappa^{\prime}-1}\|f\|_{L_{p, \alpha}(Q)}
$$

for all $\varepsilon \in\left(0, h_{0}\right)$, where $c$ does not depend on $f$ and $\varepsilon$.

Proof. Since $Q$ is bounded we have $f \in W_{p, \alpha^{\prime}}^{\bar{l}}(Q)$ and

$$
\|f\|_{W_{p, \alpha^{\prime}}^{\bar{l}}(Q)} \leq c\|f\|_{W_{p, \alpha}^{\bar{\nu}}(Q)} .
$$

Using Theorem 1 we obtain (2.26). This concludes the proof.

The results of this paper, especially Corollary 2, are necessary for the proof of the existence of global regular special solutions to Navier-Stokes equations (see [5]). 
3. Imbedding theorems for $p=q$. First we prove

TheOrem 3.1. Assume that $f \in W_{p, \alpha}^{\bar{l}}(Q), Q \subset \mathbb{E}^{n+1}$ and $Q$ satisfies the $R\left(\bar{l}, h_{0}\right)$-horn condition, $\bar{l}=\left(l_{0}, l_{1}, \ldots, l_{n}\right), 1<p<\infty, \alpha, \beta \in \mathbb{R}_{+}, \alpha \geq \beta$, $0<l_{i} \in \mathbb{Z}, 0 \leq \nu_{i} \in \mathbb{Z}, i=0,1, \ldots, n, l_{1}=l_{2}=l_{*}$ and

$$
\varkappa \equiv 1-\sum_{i=0}^{n} \frac{\nu_{i}}{l_{i}}-\frac{1}{l_{*}}(\alpha-\beta) \geq 0 .
$$

Then $D^{\bar{\nu}} f \in L_{p, \beta}(Q)$ and

$$
\left\|D^{\bar{\nu}} f\right\|_{L_{p, \beta}(Q)} \leq c_{1} h^{\varkappa}\|f\|_{L_{p, \alpha}^{\bar{l}}(Q)}+c_{2} h^{\varkappa-1}\|f\|_{L_{p, \alpha}(Q)},
$$

where $c_{1}, c_{2}$ do not depend on $f$ and $h \in\left(0, h_{0}\right), h_{0}=h_{0}(Q)$.

Proof. We consider the case $\alpha-\beta=k+\gamma, k \in \mathbb{N}_{0}, \gamma \in[0,1)$.

First we examine the case $\varkappa=0$ and $\gamma=0$. Then we write (2.6) in the form

$$
\begin{aligned}
D^{\bar{\nu}} f_{\varepsilon}(\bar{x})= & c \int_{\mathbb{E}^{n+1}} \phi_{*}^{(\bar{\nu}(k))}\left(\bar{y} / r^{\bar{\sigma}}\right) F(\bar{x}+\bar{y}) d \bar{y} \\
& +c \int_{\varepsilon}^{r} d h \sum_{i=0}^{n} \int_{\mathbb{E}^{n+1}} h^{-1-|\bar{\sigma}|} \Phi_{i}^{(\bar{\nu}(k))}\left(\bar{y} / h^{\bar{\sigma}}\right) F_{i}(\bar{x}+\bar{y}) d \bar{y}
\end{aligned}
$$

Estimating (3.3) implies

$$
\begin{aligned}
& \left\|D^{\bar{\nu}} f_{\varepsilon}\right\|_{L_{p, \beta}(Q)} \leq c\left\|\int_{\mathbb{E}^{n+1}} \Phi_{*}^{(\bar{\nu}(k))}\left(\bar{y} / r^{\bar{\sigma}}\right) F(\bar{x}+\bar{y}) d \bar{y}\right\|_{L_{p, \beta}(Q)} \\
& +c \sum_{i=0}^{n}\left\|\int_{\varepsilon}^{r} d h \int_{\mathbb{E}^{n+1}} h^{-1-|\bar{\sigma}|} \Phi_{i}^{(\bar{\nu}(k))}\left(\bar{y} / h^{\bar{\sigma}}\right) F_{i}(\bar{x}+\bar{y}) d \bar{y}\right\|_{L_{p, \beta}(Q)} \equiv M_{0}+M_{1} .
\end{aligned}
$$

Using estimates for integral operators we have

$$
M_{0} \leq c\|f\|_{L_{p, \alpha}(Q)} .
$$

Next we examine

$$
\begin{array}{r}
M_{1} \leq c \sum_{i=0}^{n}\left\|\int_{\varepsilon}^{r} h^{-1-|\bar{\sigma}|} \Phi_{i}^{(\bar{\nu}(k))}\left(\bar{y} / h^{\bar{\sigma}}\right) F_{i}^{\prime}(\bar{x}+\bar{y}) d \bar{y} d h\right\|_{L_{p}(Q)} \\
+c \sum_{i=0}^{n}\left\|\int_{\varepsilon}^{r} h^{-1-|\bar{\sigma}|} \Phi_{i}^{(\bar{\nu}(k))}\left(\bar{y} / h^{\bar{\sigma}}\right) \frac{\left|x^{\prime}\right|^{\beta}-\left|x^{\prime}+y^{\prime}\right|^{\alpha-k}}{\left|x^{\prime}+y^{\prime}\right|^{\alpha-k}} F_{i}^{\prime}(\bar{x}+\bar{y}) d \bar{y} d h\right\|_{L_{p}(Q)} \\
\equiv M_{3}+M_{4},
\end{array}
$$

where $F_{i}^{\prime}(x)=\left|x^{\prime}\right|^{\alpha-k} F_{i}(x)$.

Lemma 1.1 and the Hardy inequality (1.9) yield

$$
M_{3} \leq c \sum_{i=0}^{n}\left\|F_{i}^{\prime}\right\|_{L_{p}(Q)} \leq c \sum_{i=0}^{n}\left\|D_{i}^{l_{i}} f\right\|_{L_{p, \alpha}(Q)} .
$$


Using (1.8) in $M_{4}$ implies

$$
\begin{aligned}
& M_{4} \leq c \sum_{i=0}^{n} \| \int_{\mathbb{E}^{n+1}} \chi\left(y, R\left(\bar{l}, h_{0}\right)\right)\left(\sum_{j=0}^{n}\left|y_{j}\right|^{l_{j}}\right)^{-|\bar{\sigma}|} \\
& \times\left|\frac{\left|x^{\prime}\right|^{\beta}}{\left|x^{\prime}+y^{\prime}\right|^{\alpha-k}}-1\right| F_{i}^{\prime}(\bar{x}+\bar{y}) d \bar{y} \|_{L_{p}(Q)} \equiv M_{5} .
\end{aligned}
$$

Applying the one-dimensional Young inequality

$$
\|f * g\|_{L_{p}} \leq\|g\|_{L_{1}}\|f\|_{L_{p}}
$$

to $M_{5}$ with respect to the variables $x_{0}, x_{3}, \ldots, x_{n}$, we obtain

$$
\begin{aligned}
& M_{5} \leq c \sum_{i=0}^{n} \| \int_{\mathbb{E}^{2}} \chi\left(y^{\prime}, R\left(l_{*}, h_{0}\right)\right)\left|y^{\prime}\right|^{-2} \\
& \times\left|\frac{\left|x^{\prime}\right|^{\beta}}{\left|x^{\prime}+y^{\prime}\right|^{\alpha-k}}-1\right|\left\|F_{i}^{\prime}\left(x^{\prime}+y^{\prime}\right)\right\|_{L_{p}\left(\mathbb{E}^{n-1}\right)} d y^{\prime} \|_{L_{p}(Q)} \\
& \leq c \sum_{i=0}^{n} \| \int_{\mathbb{E}^{2}} \chi\left(y^{\prime}-x^{\prime},\right.\left.R\left(l_{*}, h_{0}\right)\right)\left|x^{\prime}-y^{\prime}\right|^{-2} \\
& \times\left|\frac{\left|x^{\prime}\right|^{\beta}}{\left|y^{\prime}\right|^{\alpha-k}}-1\right|\left\|F_{i}^{\prime}\left(y^{\prime}\right)\right\|_{L_{p}\left(\mathbb{E}^{n-1}\right)} d y^{\prime} \|_{L_{p}(Q)} \equiv M_{6} .
\end{aligned}
$$

Introducing the polar coordinates $x^{\prime}=\left(\varrho \cos \varphi_{x}, \varrho \sin \varphi_{x}\right), y^{\prime}=\left(\eta \cos \varphi_{y}\right.$, $\left.\eta \sin \varphi_{y}\right)$ we obtain

$$
\begin{aligned}
& M_{6} \leq c \sum_{i=0}^{n}\left(\int_{0}^{\infty} \varrho d \varrho \int_{0}^{2 \pi} d \varphi_{x} \mid\right. \int_{\varrho}^{\infty} \eta d \eta \int_{0}^{2 \pi} d \varphi_{y} \frac{1}{\varrho^{2}+\eta^{2}-2 \varrho \eta \cos \left(\varphi_{x}-\varphi_{y}\right)} \\
&\left.\times\left.\left|\frac{\varrho^{\beta}}{\eta^{\alpha-k}}-1\right| \bar{F}_{i}^{\prime}\left(\eta \cos \varphi_{y}, \eta \sin \varphi_{y}\right)\right|^{p}\right)^{1 / p} \equiv M_{7},
\end{aligned}
$$

where $\bar{F}_{i}^{\prime}=\left\|F_{i}^{\prime}\left(y^{\prime}\right)\right\|_{L_{p}\left(\mathbb{E}^{n-1}\right)}$.

Using the Young inequality (3.5) with respect to $\varphi_{x}$ and the expression

$$
\int_{-\pi}^{\pi} \frac{d \varphi}{\varrho^{2}+\eta^{2}-2 \varrho \eta \cos \varphi}=\frac{2 \pi}{\varrho^{2}-\eta^{2}}
$$

we obtain

$$
M_{7} \leq c \sum_{i=0}^{n}\left(\int_{0}^{\infty} \varrho d \varrho\left|\int_{\varrho}^{\infty} \frac{1}{\varrho^{2}-\eta^{2} \mid}\right| 1-\frac{\varrho^{\beta}}{\eta^{\alpha-k}}\left|\widetilde{F}_{i}^{\prime}(\eta) \eta d \eta\right|^{p}\right)^{1 / p} \equiv M_{8},
$$

where $\widetilde{F}_{i}^{\prime}(\eta)=\left(\int_{0}^{2 \pi}\left|\bar{F}_{i}^{\prime}\left(\eta \cos \varphi_{y}, \eta \sin \varphi_{y}\right)\right|^{p} d \varphi_{y}\right)^{1 / p}$. 
Introducing a new variable $\lambda$ by $\eta=\lambda \varrho$ in the inner integral in $M_{8}$ and using the generalized Minkowski inequality (see [1, Ch. 1]) we obtain

$$
\begin{aligned}
M_{8} & \leq c \int_{1}^{\infty} \frac{\lambda^{1-2 / p}}{(\lambda+1)(\lambda-1)}\left(1-\lambda^{-(\alpha-k)}\right) d \lambda \sum_{i=0}^{n}\left\|F_{i}^{\prime}\right\|_{L_{p}\left(\mathbb{E}^{n+1}\right)} \\
& \leq c \sum_{i=0}^{n}\left\|D_{i}^{l_{i}} f\right\|_{L_{p, \alpha}\left(\mathbb{E}^{n+1}\right)}
\end{aligned}
$$

where in the last inequality the Hardy inequality (1.9) was also used.

Let us consider the case $\varkappa=0$ and $\alpha-\beta=k+\gamma, \gamma>0$. Then $M_{1}$ takes the form

$$
M_{1}^{\prime}=c \sum_{i=0}^{n}\left\|\int_{\varepsilon}^{r} d h \int_{\mathbb{E}^{n+1}} h^{-1-|\bar{\sigma}|+\gamma / l_{*}} \Phi_{i}^{(\bar{\nu}(k))}\left(\bar{y} / h^{\bar{\sigma}}\right) F_{i}(\bar{x}+\bar{y}) d \bar{y}\right\|_{L_{p, \beta}(Q)} .
$$

In view of (1.8) we have

$$
\begin{aligned}
& M_{1}^{\prime} \leq c \sum_{i=0}^{n} \| \int_{\mathbb{E}^{n+1}} \chi\left(y, R\left(\bar{l}, h_{0}\right)\right)\left(\sum_{j=0}^{n}\left|y_{j}\right|^{l_{j}}\right)^{-|\bar{\sigma}|+\gamma / l_{*}} \\
& \times \frac{\left|x^{\prime}\right|^{\beta}}{\left|x^{\prime}+y^{\prime}\right|^{\alpha-k}} F_{i}^{\prime}(\bar{x}+\bar{y}) d \bar{y} \|_{L_{p}(Q)} \equiv M_{2}^{\prime} .
\end{aligned}
$$

Applying the one-dimensional Young inequality (3.4) with respect to $x_{0}, x_{3}$, $\ldots, x_{n}$ gives

$$
\begin{aligned}
M_{2}^{\prime} \leq c \sum_{i=0}^{n} \| \int_{\mathbb{E}^{2}} & \chi\left(y^{\prime}, R\left(l_{*}, h_{0}\right)\right)\left|y^{\prime}\right|^{-2+\gamma} \\
& \times \frac{\left|x^{\prime}\right|^{\beta}}{\left|x^{\prime}+y^{\prime}\right|^{\alpha-k}}\left\|F_{i}^{\prime}\left(x^{\prime}+y^{\prime}\right)\right\|_{L_{p}\left(\mathbb{E}^{n-1}\right)} d y^{\prime} \|_{L_{p}\left(\mathbb{E}^{2}\right)} \equiv M_{3}^{\prime} .
\end{aligned}
$$

Changing variables implies

$$
M_{3}^{\prime}=c \sum_{i=0}^{n}\left\|\int_{\mathbb{E}^{2}} \chi\left(y^{\prime}-x^{\prime}, R\left(l_{*}, h_{0}\right)\right)\left|x^{\prime}-y^{\prime}\right|^{-2+\gamma} \frac{\left|x^{\prime}\right|^{\beta}}{\left|y^{\prime}\right|^{\alpha-k}} \bar{F}_{i}^{\prime}\left(y^{\prime}\right) d y^{\prime}\right\|_{L_{p}\left(\mathbb{E}^{2}\right)},
$$

where $\bar{F}_{i}^{\prime}\left(y^{\prime}\right)=\left\|F_{i}^{\prime}\left(y^{\prime}\right)\right\|_{L_{p}\left(\mathbb{E}^{n-1}\right)}$. Introducing the polar coordinates $x^{\prime}=$ $\left(\varrho \cos \varphi_{x}, \varrho \sin \varphi_{x}\right), y^{\prime}=\left(\eta \cos \varphi^{\prime}, \eta \sin \varphi^{\prime}\right), \varphi=\varphi_{x}-\varphi^{\prime}$, in $M_{3}^{\prime}$ yields

$$
\begin{aligned}
M_{3}^{\prime} \leq c \sum_{i=0}^{n}\left(\int_{0}^{\infty} \varrho d \varrho \int_{0}^{2 \pi} d \varphi^{\prime} \mid \int_{\varrho}^{\infty} \eta d \eta \int_{0}^{2 \pi} \frac{d \varphi_{x}}{\left(\varrho^{2}+\eta^{2}-2 \varrho \eta \cos \left(\varphi_{x}-\varphi^{\prime}\right)\right)^{1-\gamma / 2}}\right. \\
\left.\times\left.\frac{\varrho^{\beta}}{\eta^{\alpha-k}} F_{i}^{\prime}\left(\eta \cos \varphi^{\prime}, \eta \sin \varphi^{\prime}\right)\right|^{p}\right)^{1 / p} \equiv M_{4}^{\prime} .
\end{aligned}
$$


Using the fact that the integral over $\varphi_{x}$ can be made independent of $\varphi^{\prime}$ and then applying the Minkowski inequality (see [1, Ch. 1]) we obtain

$$
\begin{aligned}
M_{4}^{\prime} \leq c \sum_{i=0}^{n}\left(\int_{0}^{\infty} \varrho d \varrho\right. & \mid \int_{\varrho}^{\infty} \eta d \eta \int_{0}^{2 \pi} \frac{d \varphi}{\left(\varrho^{2}+\eta^{2}-2 \varrho \eta \cos \varphi\right)^{1-\gamma / 2}} \frac{\varrho^{\beta}}{\eta^{\alpha-k}} \\
& \left.\times\left.\left(\int_{0}^{2 \pi}\left|F_{i}^{\prime}\left(\eta \cos \varphi^{\prime}, \eta \sin \varphi^{\prime}\right)\right|^{p} d \varphi^{\prime}\right)^{1 / p}\right|^{p}\right)^{1 / p} \equiv M_{5}^{\prime} .
\end{aligned}
$$

Changing variables $\eta=\lambda \varrho$ gives

$$
\begin{aligned}
M_{5}^{\prime}=c \sum_{i=0}^{n}\left(\int_{0}^{\infty} \varrho d \varrho \mid \int_{1}^{\infty}\right. & \lambda d \lambda \int_{0}^{2 \pi} \frac{d \varphi}{\left(\lambda^{2}+1-2 \lambda \cos \varphi\right)^{1-\gamma / 2}} \lambda^{-(\alpha-k)} \\
& \left.\times\left.\left(\int_{0}^{2 \pi}\left|F_{i}^{\prime}\left(\lambda \varrho \cos \varphi^{\prime}, \lambda \varrho \sin \varphi^{\prime}\right)\right|^{p} d \varphi^{\prime}\right)^{1 / p}\right|^{p}\right)^{1 / p} .
\end{aligned}
$$

Applying the Minkowski inequality (see [1, Ch. 1]) yields

$$
\begin{aligned}
M_{5}^{\prime} \leq c \sum_{i=0}^{n} \int_{1}^{\infty} \lambda d \lambda & \int_{0}^{2 \pi} \frac{d \varphi}{\left(\lambda^{2}+1-2 \lambda \cos \varphi\right)^{1-\gamma / 2}} \lambda^{-(\alpha-k)} \\
& \times\left(\int_{0}^{\infty} \varrho d \varrho \int_{0}^{2 \pi} d \varphi^{\prime}\left|F_{i}^{\prime}\left(\lambda \varrho \cos \varphi^{\prime}, \lambda \varrho \sin \varphi^{\prime}\right)\right|^{p}\right)^{1 / p} \equiv M_{6}^{\prime} .
\end{aligned}
$$

Introducing a new variable $\sigma=\lambda \varrho, d \sigma=\lambda d \varrho$, implies

$$
\begin{aligned}
M_{6}^{\prime}= & c \sum_{i=0}^{n} \int_{1}^{\infty} \lambda d \lambda \int_{0}^{2 \pi} \frac{d \varphi}{\left(\lambda^{2}+1-2 \lambda \cos \varphi\right)^{1-\gamma / 2}} \lambda^{-(\alpha-k)-2 / p} \\
& \times\left(\int_{0}^{\infty} \sigma d \sigma \int_{0}^{2 \pi} d \varphi^{\prime}\left|F_{i}^{\prime}\left(\sigma \cos \varphi^{\prime}, \sigma \sin \varphi^{\prime}\right)\right|^{p}\right)^{1 / p} \\
\leq c & \sum_{i=0}^{n} \int_{1}^{\infty} \lambda d \lambda \int_{0}^{2 \pi} \frac{d \varphi}{\left(\lambda^{2}+1-2 \lambda \cos \varphi\right)^{1-\gamma / 2}} \lambda^{-(\alpha-k)-2 / p}\left\|F_{i}\right\|_{L_{p}\left(\mathbb{E}^{n+1}\right)} \equiv M_{7}^{\prime} .
\end{aligned}
$$

Passing to the Cartesian coordinates $z_{1}=\lambda \cos \varphi, z_{2}=\lambda \sin \varphi, \bar{z}_{0}=(1,0)$, $\bar{z}=\left(z_{1}, z_{2}\right)$ we write $M_{7}^{\prime}$ in the form

$$
M_{7}^{\prime}=c \sum_{i=0}^{n}\left\|F_{i}\right\|_{L_{p}\left(\mathbb{E}^{n+1}\right)} \int_{|\bar{z}| \geq 1} d \bar{z} \frac{|\bar{z}|^{-(\alpha-k)-2 / p}}{\left|\bar{z}-\bar{z}_{0}\right|^{2-\gamma}} .
$$

For $|\bar{z}|$ large the integral converges for $\alpha-k+2 / p>\gamma$. 
For $|\bar{z}|$ in a neighbourhood of 1 we can show convergence by passing to the coordinates with origin at $\bar{z}_{0}$. Finally we have

$$
M_{7}^{\prime} \leq c \sum_{i=0}^{n}\left\|F_{i}\right\|_{L_{p}\left(\mathbb{E}^{n+1}\right)} .
$$

Applying now the remarks from [3] we can let $\varepsilon \rightarrow 0$ to obtain (3.2).

Let $\varkappa>0$. Using the considerations from [1, Ch. 3] we obtain (3.2) with a parameter $\delta>0$.

For $Q$ bounded we apply the standard considerations with a partition of unity. This concludes the proof.

\section{References}

[1] O. V. Besov, V. P. Il'in and S. M. Nikol'skiı̆, Integral Representations of Functions and Imbedding Theorems, Nauka, Moscow, 1975 (in Russian).

[2] V. P. Il'in, Some integral inequalities and applications in the theory of differentiable functions of several variables, Mat. Sb. 54 (1961), 331-380 (in Russian).

[3] A. F. Kocharli, Some weighted imbedding theorems for domains with nonsmooth boundary, Trudy Mat. Inst. Steklov. 131 (1974), 128-146 (in Russian).

[4] W. M. Zajączkowski, On theorem of embedding for weighted Sobolev spaces, Bull. Polish Acad. Sci. Math. 33 (1985), 115-121.

[5] - On global special solutions to Navier-Stokes equations with boundary slip conditions in a cylindrical domain. Existence, preprint 616, Inst. Math., Polish Acad. Sci., 2001 .

Institute of Mathematics

Polish Academy of Sciences

Śniadeckich 8

00-950 Warszawa, Poland

E-mail: wz@impan.gov.pl
Institute of Mathematics and Operations Research Military University of Technology

S. Kaliskiego 2

00-908 Warszawa, Poland

Received on 12.10.2000;

revised version on \%.11.2001 